\title{
Intelligent Entity Coordinator
}

\author{
Kritika \\ Assistant Professor \\ BPIT, GGSIPU \\ Delhi, India
}

\author{
Gagan Upadhyay \\ Student (CSE) \\ BPIT, GGSIPU \\ Delhi, India
}

\author{
Tushar \\ Student (CSE) \\ BPIT, GGSIPU \\ Delhi, India
}

\author{
Shubham \\ Student (CSE) \\ BPIT, GGSIPU \\ Delhi, India
}

\begin{abstract}
Today everyone is living in a world that is dominated by technology. To be able to develop a product using the current technology that will be helpful to various people is a big contribution to the society. This research paper represents the idea, design and implementation of a low cost but yet easily accessible android based Entity Coordinator system. The purpose of this project is to build an Intelligent Entity Coordinator to control switching of home appliances from any part of the world having an internet access. This will lead to less consumption of energy in case a person forgets to turn off their home appliances. As we know that, electricity is precious and should be used in an efficient way, this project will help others to build an efficient habit towards the electricity conservation. The design is based on Node MCU board and the home appliances are connected to the input/ output ports of this board via relays. The communication between the Android device and the Node MCU board is through Cloud Mqtt. This system is developed to be low cost and scalable allowing variety of devices to be controlled with minimum changes to its core. Also, the artificial intelligence is used for the user interaction better.
\end{abstract}

\section{General Terms}

Publisher-Subscriber Model

\section{Keywords}

Iot, Mqtt, Android, Cloud Mqtt, Energy Conservation

\section{INTRODUCTION}

Internet based technologies are becoming very popular around the world and the users want the wireless lifestyle which enables them to have access to everything from anywhere in the world. Turning on/off electrical appliances of office/house is an easy task for the human beings. But, it comes out to be a very physical task because we have to physically walk towards the switches and then only we can control our appliances. To accomplish this task simply and quickly, an Intelligent Entity Coordinator is developed, that is a model based on automating the appliances of our house at a very cheap rate. An android app was built including an interactive UI of app that helps clients to control the devices that are connected to internet with the help of IOT device. In day to day life, people forget to turn off the appliances of their house, so this system is very much helpful in reminding them that the appliances of their house are still in the running mode and they should conserve their energy sources by turning off the appliances and hence save the electricity which could suppress emission of $\mathrm{CO}_{2}$ in the environment [1].

This model is a very cost effective model as the cost of the hardware used in this model is such that even a person belonging to an average middle class can afford. The cloud based system is completely free of cost. Cloud Mqtt server provides a free access to the cloud database. Hence, in studies, it has been found that most of the working people forget to turn off their appliances while leaving to work. This project will help those people in need. This project is feasible, efficient, reliable, cost effective, revolutionary, eco-friendly and can be easily operated.

The "Entity Coordinator" concept is being used from many years. The terms "Smart Homes", "Intelligent Homes" are being used synonymous to each other and were used to introduce the concept of networking, smart appliances and smart devices in the house. Intelligent Entity Coordinator represents a great opportunity for creating new fields in engineering related to Hardware, and Computing. It includes controlling of lighting, appliances, and other systems, to provide improved comfort, energy efficiency and security to houses.

In the present time, the technologically advanced world is getting more and more advance as new technology is emerging day by day into the lives of human beings even in their houses as well.

Intelligent Entity Coordinator system is reaching all over the world. The system is used to control and monitor electronic security systems, lighting, climate, appliances any equipment, etc. This system could be used to make houses smarter in such a way that people can interact with their house as it could be controlled from a single human touch from a Smartphone. The benefits of this system are that it is secure and saves money, time, maintenance costs, and makes life easier.

The basic idea of this project is to save money because it is a cost effective model i.e. the hardware is cheap as compared to other models and the cloud mqtt server provides a free cloud service to every individual, having an account on Cloud Mqtt, it is also free of cost.

\section{LITERATURE SURVEY}

At present appliances are connected with internet using Arduino which is costly as well as slightly tough to implement with respect to the proposed work. Bluetooth [2], Infrared and ZigBee technologies are used as communicating medium between various appliances and humans. An automated home could be based on a platform or hubs that control internet operated devices and appliances. For instance, using Apple's HomeKit, producers can get their house products and accessories be controlled by an application in iOS devices such as the iPhone and the Apple Smart Watch. This can be a dedicated app or iOS original applications such as Siri. This can be represented in the case of Lenovo's Smart Home Essentials, which is a series of smart home devices that are coordinated through Apple's Home app or Siri without the requirement for a Wi-Fi bridge. Numerous research work has done, which proves that MQTT is better than HTTP for the nodes with constrained resources. It has been proven that data transmission through MQTT consumes only about $0.05 \%$ of battery/hour by using 3G for network connectivity [3] and a research [4] shows that a prototype is made to control smart 
entities using SMS. GSM network and the devices are bridged using a micro-controller.

\section{HARDWARE IMPLEMENTATION}

NodeMCU is a Wi-Fi module developed by Espressif Systems. It is based on ESP8266 -12E Wi-Fi module. It is an integrated chip designed to provide internet connectivity in a tiny package

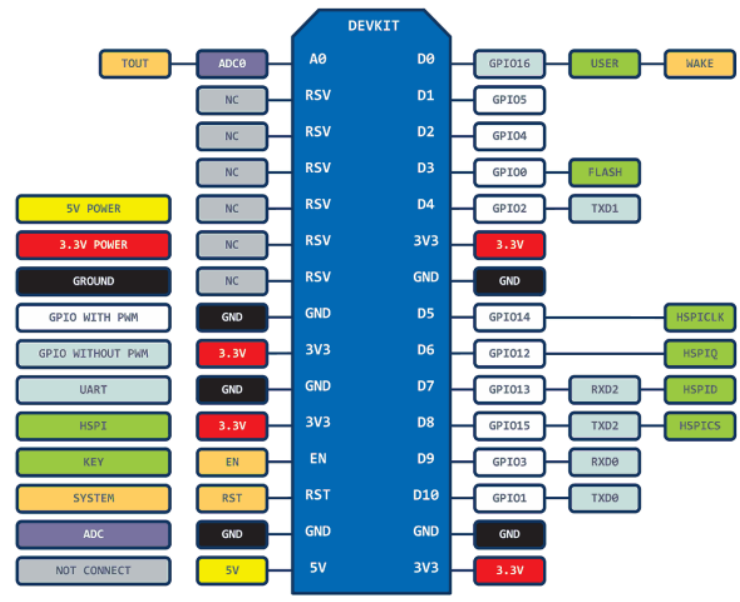

Figure 1: NODE MCU Pin Diagram [5]

It can be programmed directly through USB port using LUA, $\mathrm{C}++$ programming using Platform IO IDE in VS Code Editor. By simple programming, a Wi-Fi connection can be established and input/output pins can be defined according to the needs, connecting it to a cloud web server and a lot more. NodeMCU is a module that can be connected to Wi-Fi. It combines the features of $\mathrm{Wi}-\mathrm{Fi}$ access point and station, microcontroller. These features make the NodeMCU more powerful tool for Wi-Fi networking. It could be used as an access point or station, connect to a cloud web server or connect to internet to fetch and upload data.

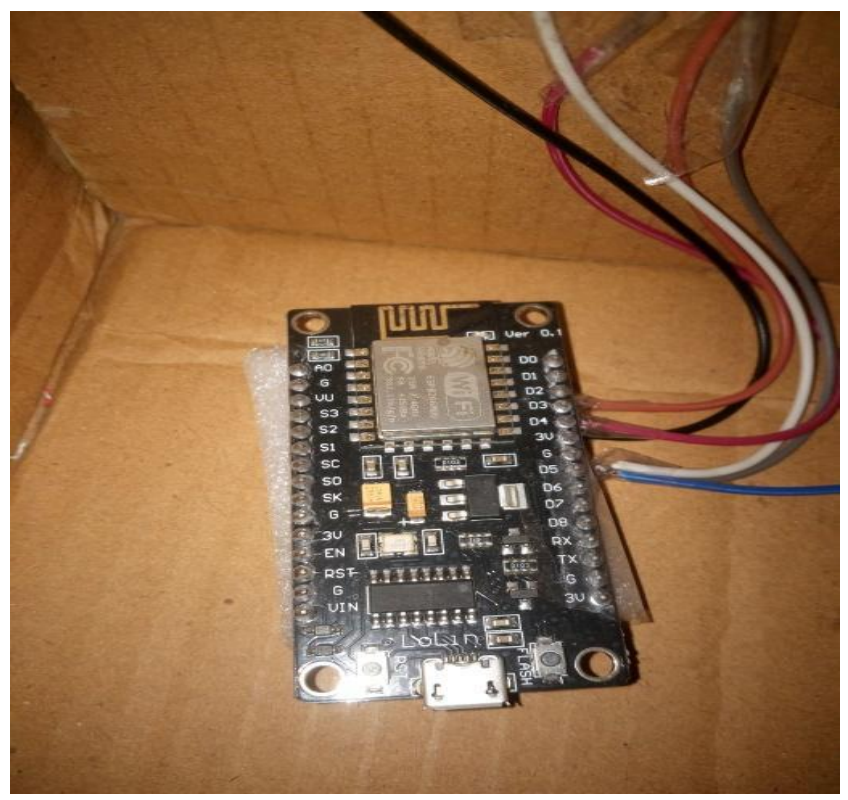

Figure 2: NODE MCU Chip
As Arduino began developing new MCU boards based on non-AVR processors like the ARM and SAM MCU and used in the Arduino Due, they require to modify the Arduino IDE so that it could be easy to change the IDE to support alternative tool chains to allow Arduino $\mathrm{C} / \mathrm{C}++$ to be compiled to these new processors. This was done with the introduction of the Board Manager and the SAM Core. A "core" is a collection of software components required by the Board Manager and the Arduino IDE to compile an Arduino $\mathrm{C} / \mathrm{C}++$ source file to the target MCU's machine language. Some ESP8266 enthusiasts have developed an Arduino core for the ESP8266 WiFi SoC that is available at the GitHub ESP8266 Core webpage. This is popularly called the "ESP8266 Core for the Arduino IDE" and it has become one of the leading software development platforms for the various ESP8266 based modules and development boards, including Node MCUs.

\section{SOFTWARE IMPLEMENTATION 4.1 Internet of Things}

The internet of things (Iot) is a computing concept that describes the idea of connecting objects being to the internet that are in a daily use and being able to interact to other devices.

Iot is an approach of communication between non-living things and living things. Devices can communicate with each other with the help of an internet connection. It may include devices such as Light, Fan, Television, Refrigerator, etc.

The Iot is important because an object that can represent itself digitally becomes something greater than the object by itself. The objects are now connected to surrounding objects and have access to cloud database. The devices can now send and receive data from cloud mqtt. When many objects act in resonance, they are termed as having "ambient intelligence."

\subsection{Android}

Android is a mobile operating system (OS) first developed by a Silicon Valley company by the name of Android Inc. Android is an operating system that acts as a mediocre between living things and non-living things. An android app was built including an interactive UI of app that helps clients to control the devices that are connected to internet with the help of Iot device.

\subsection{MQTT}

Mqtt (Message Queuing Telemetry Transport) is an ISO Standard; Machine-to-Machine based Protocol for connectivity of Iot devices. It is a publisher-subscriber based messaging protocol that is used for the interaction of various devices using Iot.

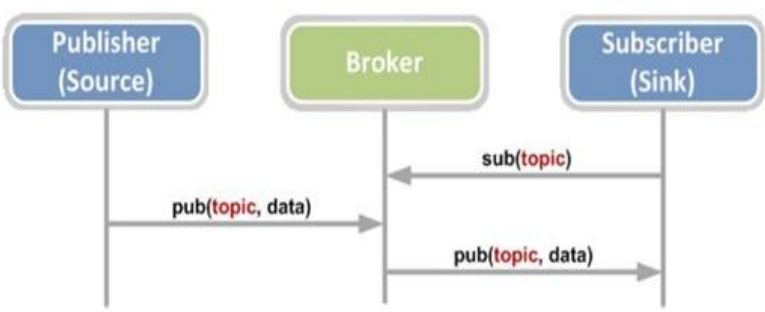

Figure 3: The Publisher-Subscriber Model [6] 


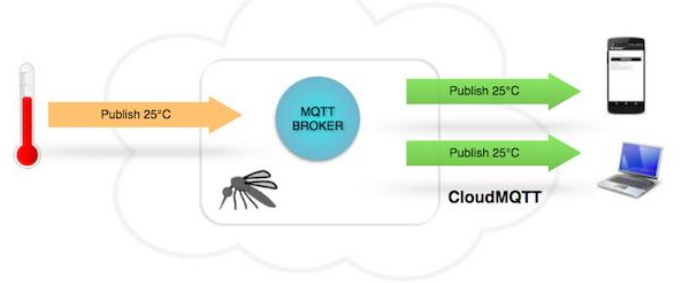

Figure 4: Visualization Of Publisher-Subscriber Model[7]

Publisher-Subscriber model means that the devices, appliances can publish the message and can receive the message sent by another device which is a subscriber to that protocol. It works over TCP/IP i.e. Transmission Control Protocol/Internet Protocol that is the protocol that works on.

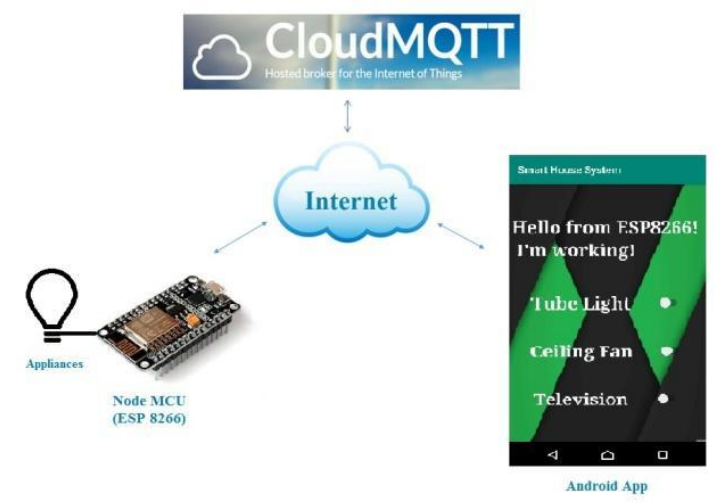

Figure 5: Functionality of Project

When the NODE MCU chip is turned on it subscribes the mqtt cloud. Intelligent Entity Coordinator takes command from the android app, with the help of internet connection it sends the request to the cloud. The mqtt cloud now publishes the message to the NODE MCU Chip and the chip acts accordingly.

\section{RESULT AND COMPARISON}

Table 1. Comparison of various technologies

\begin{tabular}{|c|c|c|c|}
\hline \multirow{2}{*}{ S.No. } & \multicolumn{3}{|c|}{ Range of Technologies } \\
\cline { 2 - 4 } & Technology & Range (in m) & Type Of Waves \\
\hline 1. & Infrared & 10 & Light Waves \\
\hline 2. & Bluetooth & 100 & Radio Waves \\
\hline 3. & Internet & Worldwide & Radio Waves \\
\hline
\end{tabular}

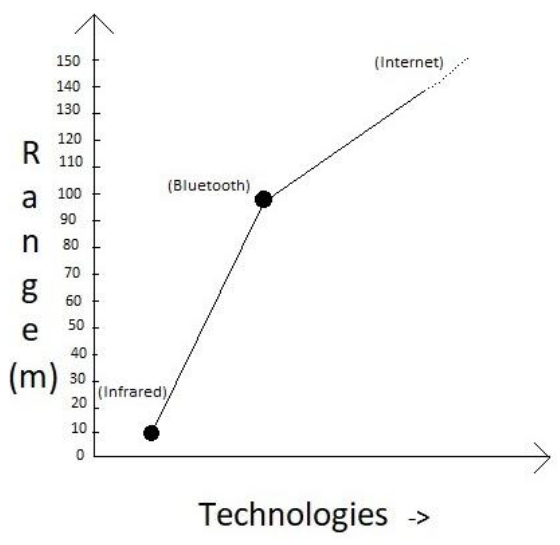

On comparing Internet with Bluetooth and Infrared, it is clear that the Internet is better from the rest of the two technologies. Some actions performed on the app are as follows:

When TV is switched On or Off, message is displayed:
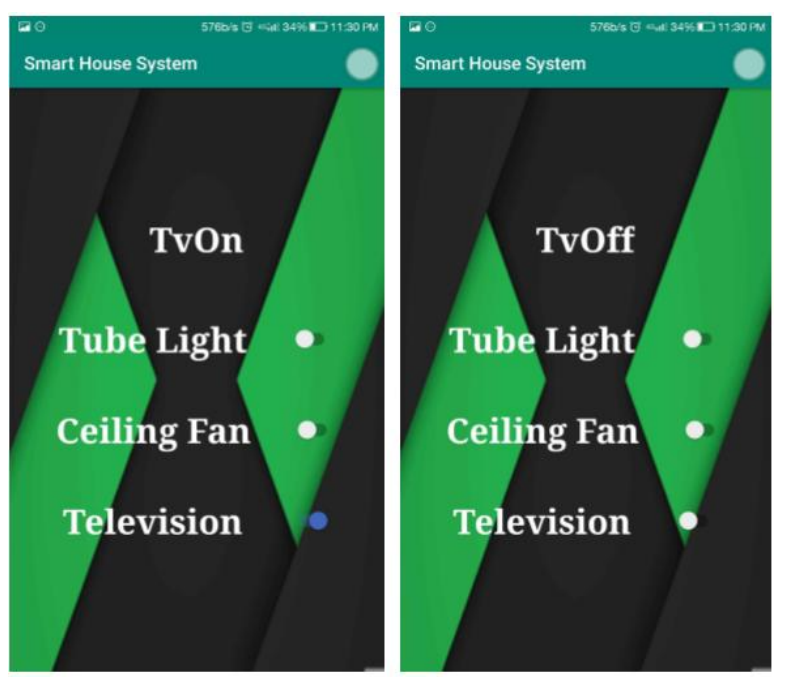

Figure 6: TV is turned on and off

When Fan is switched On or Off, message is:

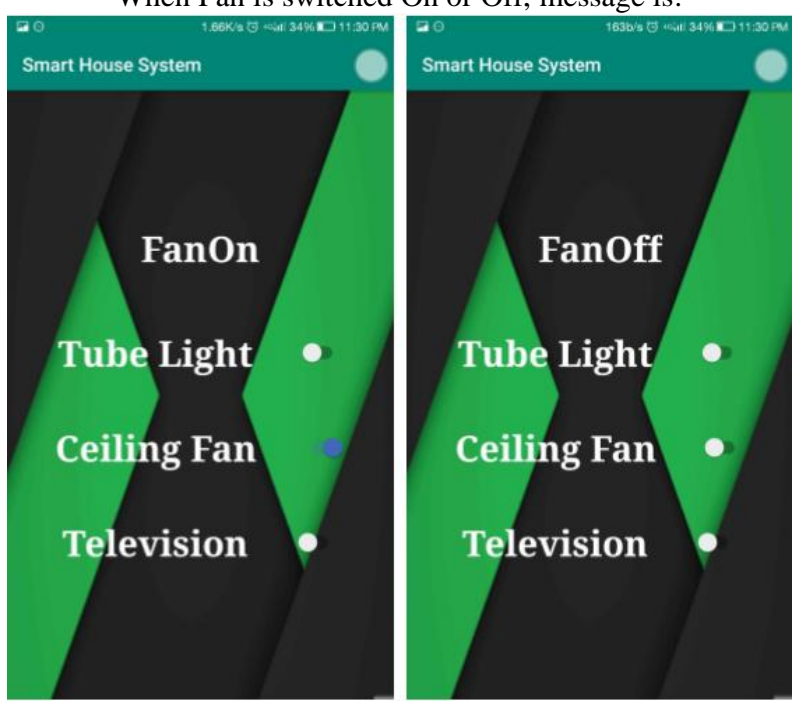

Figure 7: Fan is turned on and off 


\section{CONCLUSION}

The Entity Coordination using Internet of Things has been experimentally proven to work satisfactorily by connecting simple appliances to it and the appliances were successfully controlled through android app via internet access. The designed system monitors the appliances of the house. It also stores the data in the cloud (Cloud Mqtt) in a timely manner as it gets an internet access. This will help the user to analyze the condition of various parameters in the home anytime anywhere across the world. The project was tested to turn appliances on and off such as: Tube lights, Fans and other appliances. When the switch of the appliance is turned $\mathrm{ON}$, a message is printed on the cloud server which conveys the message to the android app, hence making it convenient to know about status of appliance.

Using this system as framework, this system can be expanded to include various other options which could include home security feature like capturing the photo of a person moving around the house and storing it onto the cloud. This would reduce the data storage that uses the CCTV camera which will record all the time and will store it. The system can be expanded for energy surveillance, or weather stations. This kind of a system with respective changes can be implemented in the hospitals for especially abled people or in radioactive industries where human invasion is impossible or harmful, and it can also be implemented for environmental monitoring.

\section{ACKNOWLEDGMENTS}

Our thanks to our mentor Ms. Kritika who have contributed towards development of this research paper.

\section{REFERENCES}

[1] https://www.sciencedaily.com/releases/2010/06/1006301 01022.htm.
[2] https://iot.electronicsforu.com/expert-opinion/wirelesstechnologies-iot/

[3] H. El Kamchouchi and A. El Shafee, "Design and prototype implementation of sms based home automation system," in Electronics Design, Systems and Applications (ICEDSA), 2012 IEEE International Conference on, Nov 2012, pp. 162-167.

[4] A. Kumar and S. Johari, "Push notification as a business enhancement technique for e-commerce," in 2015 Third International Conference on Image Information Processing (ICIIP), Dec 2015, pp. 450-454.

[5] https://researchgate.net/figure/The-pinconfiguration-of-Node-MCU_fig3_323138617

[6] https://m.eet.com/media/1171446/0912embmqtt01. png

[7] https://www.cloudmqtt.com/images/cloudmqtt_overview .png

[8] https://www.cloudmqtt.com/docs.html

[9] http://public.dhe.ibm.com/software/dw/webservices/wsmqtt/mqtt-v3r1.html

[10] http://nodemcu.com/index_en.html/

[11] https://www.espressif.com/en/products/hardware/es p8266ex/overview

[12] https://github.com/Zolertia/Resources/wiki/MQTTclient 